\title{
Effects of Magnetic Field on the Blood Flow in an Aneurismal Aorta
}

\author{
Nezar $\mathbf{M}^{1 *}$, Aggoune $\mathrm{N}^{2}$, Nezar DJ ${ }^{3}$, Abdessemed $\mathbf{R}^{4}$, Nezar $\mathrm{KS}^{5}$ and Nezar $\mathrm{A}^{5}$ \\ ${ }^{1}$ Laboratoire d'Innovation en éco-conception, construction et génie sismique (LICEC_GS), Algeria \\ ${ }^{2}$ Laboratoirede Mécanique des structures et Matériaux (LaMsM), Algeria \\ ${ }^{3}$ Laboratoire de Physique Energétique appliquée de Batna (LPEA), Algeria \\ ${ }^{4}$ Laboratoire d'Electrotechnique de Batna (LEB), Algeria \\ ${ }^{5}$ Centre hospitalier universitaire de Batna $\mathrm{CHU}$, Algeria
}

\begin{abstract}
The goal of this paper is to analyze the effect of the magnetic field, applied to a blood tissue whose walls present an aneurism arterial. The interest of this study is to evaluate the rate of influence, if there existed, of the applied magnetic field and to check the preconceived validity of the magneto therapy. The FLUENT computational fluid dynamic code is used to carry out all computation in this work, which is selected to resolve the MHD equations.
\end{abstract}

Keywords: Aneurism; Blood flow; Magnetic field; Magneto therapy; Harmful effects; CFD

\section{Introduction}

The biological fabrics are strongly heterogeneous mediums with very particular properties, and the attack of diseases as aortic aneurism became, unfortunately, very widespread, as caused by arterial hypertension; an essential characteristic of the actual environment. The most unfavorable consequences of this disease are the formation of thrombus (stone of blood); often induced by the slowness of the blood flow inside this aneurism. To mitigate this problem; a library search was undertaken; and public works by CAT and Huang, revealed that an exposed blood flow with a field of intensity 1,3 tesla, will be fluxes; in less than one minute. To check this prejudice, we made a numerical study; on the state of a blood flow of the aorta; by considering the two following principal cases:

- The study of the healthy state of the aorta, which corresponds to the application of a magnetic field in the absence of aneurism;

- The study of the aorta beginning aneurismal state, which makes it possible to ensure a good visibility of the least effects emerging following the interaction of the two phenomena [1].

\section{What an aneurism?}

An aneurism is a dilation of an artery wall, in general, of the aorta. This dilation occurs; in general; in a weak area of the arterial wall. The blood pressure involves swelling towards the outside of the weak area. If it is not treated, aneurism can break and involve an internal bleeding. Aneurisms can develop everywhere along the aorta. The most frequent cause of aortic aneurisms is the atherosclerosis (arterial hypertension, diabetes, tobacco... etc), which weaken the wall of the aorta $[2,3]$.

\section{Test bench}

To include the side effects of this problem, we considered it useful to reproduce, with real dimensions, the body (Figure 1) with a prolongation of the aorta on the side of the aneurismal zone and thus, to allow as well as possible to include the side effects of this problem, we considered it useful to reproduce, with real dimensions, the body (Figure 1) with a prolongation of the aorta on the side of the aneurismal zone and thus, to allow as well as possible to appreciate the evolution of the blood flow under the action of the two physical phenomena. The interest will be carried, in this case, with the principal channel which is "the aorta" and not with the junctions [4].

\section{Mathematical Formulation}

The theory biophysics presents blood, like, a rheofluidifiant fluid; in other words, its dynamic viscosity is described, according to the force of friction $\mathrm{F}$, which a blood layer exerts on its adjacent:

$$
F=\tau S \frac{d \vartheta}{d z}
$$

Where,

$\tau$ : Dynamic viscosity coefficient

S: Surface common to both layers $\left(\mathrm{m}^{2}\right)$

९: Rate of the blood flow

And it's kinematic viscosity is expressed by the relation:

$v=\frac{\tau}{\rho}$

Where: $\rho$ : is the density of blood $\left(\mathrm{kg} / \mathrm{m}^{3}\right)$

Consequently, its variation laws are described by the Navier Stocks equations:

- $\quad$ Equation of continuity:

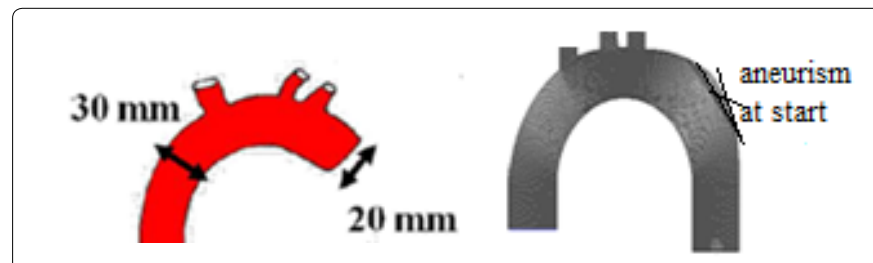

Figure 1: The dimensions of the test bench.

*Corresponding author: Nezar M, Laboratoire d'Innovation en éco-conception construction et génie sismique (LICEC_GS), Algeria, Tel: +21333230202; E-mail: m.nezar@univ-batna2.dz

Received May 16, 2017; Accepted June 03, 2017; Published June 09, 2017

Citation: Nezar M, Aggoune N, Nezar DJ, Abdessemed R, Nezar KS, et al. (2017) Effects of Magnetic Field on the Blood Flow in an Aneurismal Aorta. J Electr Electron Syst 6: 227. doi: 10.4172/2332-0796.1000227

Copyright: $\odot 2017$ Nezar M, et al. This is an open-access article distributed under the terms of the Creative Commons Attribution License, which permits unrestricted use, distribution, and reproduction in any medium, provided the original author and source are credited. 
$\frac{\partial \rho}{\partial t}+\sum_{j=1}^{3} \frac{\partial\left(\rho \bar{u}_{j}\right)}{\partial x_{j}}=0$

- Equations of momentum:

$\frac{\partial(\rho \bar{u})}{\partial t}+\sum_{j=1}^{3} \frac{\partial\left(\rho \bar{u}_{i} \bar{u}_{j}\right)}{\partial x_{j}}=-\frac{\partial \rho}{\partial x_{i}}+\sum_{j=1}^{3} \frac{\partial}{\partial x_{j}}\left[\mu\left[\frac{\partial \bar{u}_{i}}{\partial x_{j}}+\frac{\partial \bar{u}_{j}}{\partial x_{i}}-\frac{2}{3} \delta_{i j} \sum_{j=1}^{3} \frac{\partial u_{i}}{\partial x_{i}}\right]\right]+\sum_{j=1}^{3} \frac{\partial}{\partial x_{j}}\left(-\rho u_{i} u_{j}\right)+F_{v}$

Where: $\mathrm{F}_{\mathrm{v}}$ is the electromagnetic force resulting from the interaction of the induced currents density and the magnetic field which gave its birth. It is written in the form:

$$
\begin{aligned}
q & =\frac{-}{\sigma} j \cdot j \\
q & =\frac{1}{\sigma} \vec{j} \cdot \vec{j} \text { with } \vec{j}=\sigma \vec{E}\left(\mathrm{~W} / \mathrm{m}^{3}\right)
\end{aligned}
$$

$\sigma:$ is the fluid electric conductivity,

$\mathrm{q}$ : induction of the magnetic field

$\vec{E}$ : is the local electric field

$\vec{B}$ is the vector of the magnetic field

- Equation of energy:

$$
\frac{\partial(\rho E)}{\partial t}+\sum_{j=1}^{3} \frac{\partial\left(\rho E \overline{u_{j}}\right)}{\partial x_{j}}=\sum_{i=1}^{3} \sum_{j=1}^{3}\left(\frac{\partial}{\partial x_{j}}\left(\tau_{i j}-\rho \bar{u}_{i} u_{j}\right) \overline{u_{i}}\right)-\sum_{j=1}^{3} \frac{\partial}{\partial x_{j}} q_{j}+S_{E}
$$

\section{Simulation}

To try to check the harmful effect, of the magnetic fields on the healthy living organisms, would be the task of this first test. In the second, it would be necessary to check the rate of this harmfulness, in the presence of aneurism. As for the interest granted to aneurism, in particular, and the choice carried to return it the determining reference frame of these effects; it returns, with the increased probability of attack by this disease attends so much favored by the conditions of surrounding current: stress associated permanently with the magnetic field reigning.

On the basis of these reports, the digital simulation will be carried out, by respecting the data of an incompressible medium and an animated blood flow of a speed of $0.035 \mathrm{~m} / \mathrm{s}$, and this, to make it possible to approach the maximum of a steady flow. Finally, Table 1, gathers the physical properties used in simulation and the found results are given hereafter [4-8].

\section{Results and Analysis}

Healthy state: Figure 2 represents the cartography of the lines speed, which announces a state of driven back flow, with a maximum speed which reduce gradually with $0.015 \mathrm{~m} / \mathrm{s}$, while moving away from its source. As for the spectral analysis (Figure 3), it reflects a signal

\begin{tabular}{|l|c|}
\hline Property of the blood flow & Value \\
\hline Density $\rho$ & $\rho=1010 \mathrm{~kg} / \mathrm{m}^{3}$ \\
\hline Kinematic viscosity $\nu$ & $v=1.0 \times 10^{-5} \mathrm{~m}^{2} \mathrm{~s}^{-1}$ \\
\hline Dynamic viscosity $\mu$ & $\mu=0.0036 \mathrm{~Pa} . \mathrm{s}$ \\
\hline Thermal conductivity $\mathrm{k}$ & $\mathrm{k}=0.50 \mathrm{Wm}^{-1} \mathrm{~K}^{-1}$ \\
\hline Heat capacity Cp & $\mathrm{Cp}=3600 \mathrm{~J}^{-\mathrm{kg}^{-1} \mathrm{~K}^{-1}}$ \\
\hline Electric conductivity $\sigma$ & $\sigma=0.601 / \Omega \mathrm{m}$ \\
\hline Magnetic permeability $\mu_{B}$ & $\mu_{\mathrm{B}}=0.0012 \mathrm{~h} / \mathrm{m}$ \\
\hline
\end{tabular}

Table 1: Physical properties of the blood flow.

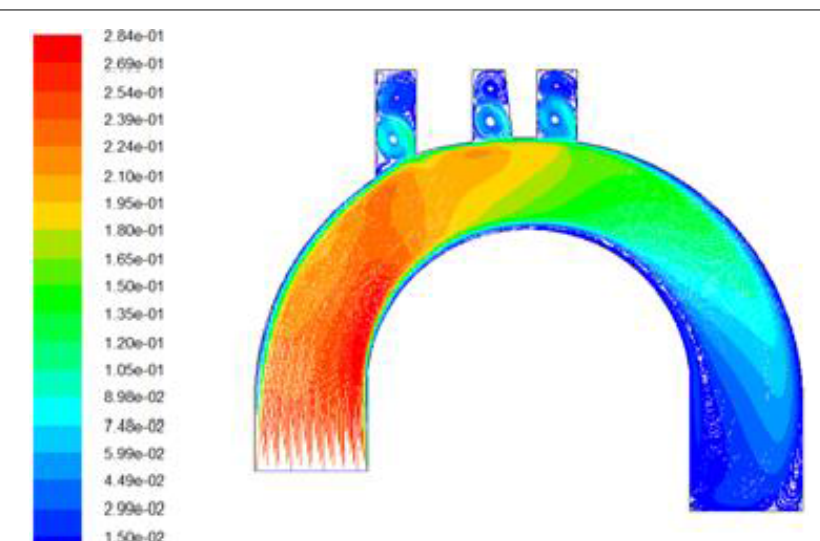

Figure 2: Velocity path lines.

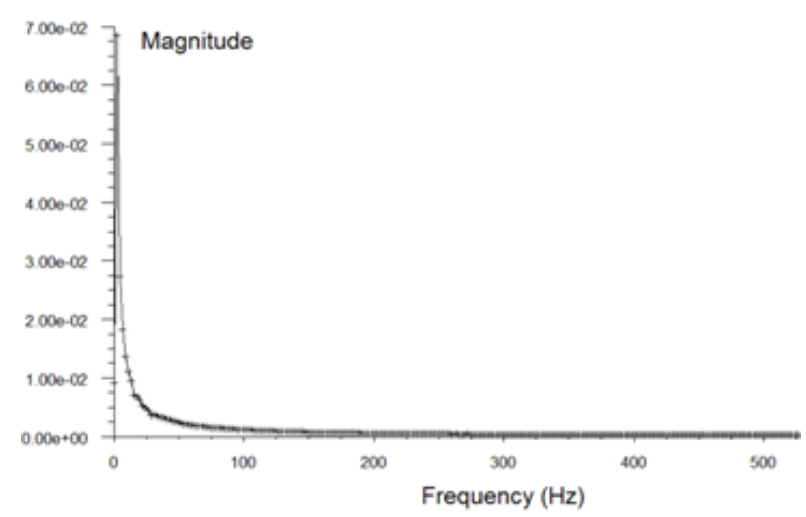

Figure 3: Velocity spectral.

evolving quickly to a mode of stable laminar flow after a peak of 0.07 of amplitude of the fundamental one.

Under these test conditions, the application of a magnetic field and the evaluation of its effects compared to the case of aneurism, are the single means of confirming the harmful effect of the latter. Also, reproduce the "Tao et Huang" test, by applying an inclined magnetic field of 1,3 Tesla, is the other approach which makes it possible to draw up a powerful comparative study.

Figures 4 and 5 are the results ensuing from this test. Its cartography shows clearly that the flow, always, takes the steady form, with almost the same data. But the Fourier spectral analysis, while illustrating, a steady behavior speed, shows a striking attenuation of the fundamental amplitude follow-up of a weak peak 0.003 disturber which can be at the origin of an incipient disorder. This result (Figure 5), can be returned to the gravitational effect and more precisely to the resultant of the two forces: like the force due to the component $\left(\mathrm{B}_{\mathrm{y}}=1.3 \sin (\pi / 4)\right)$, is in direct opposition of the force of gravity, a real physical report.

State of aneurismal disorder: In this test, only the effect of the beginning of aneurism was considered.

In the absence of magnetic field: The results (Figures 6 and 7) show clearly the appearance of a turbulent effect in the vicinity of the zone weakened by aneurism. Figure 6 , announces an unsteady speed values from the beginning to the end of the sample, presenting turbulent rollers in the aneurismal zone, this report is marked by the succession of spectra (Figure 7) of almost identical size. As for the fundamental 

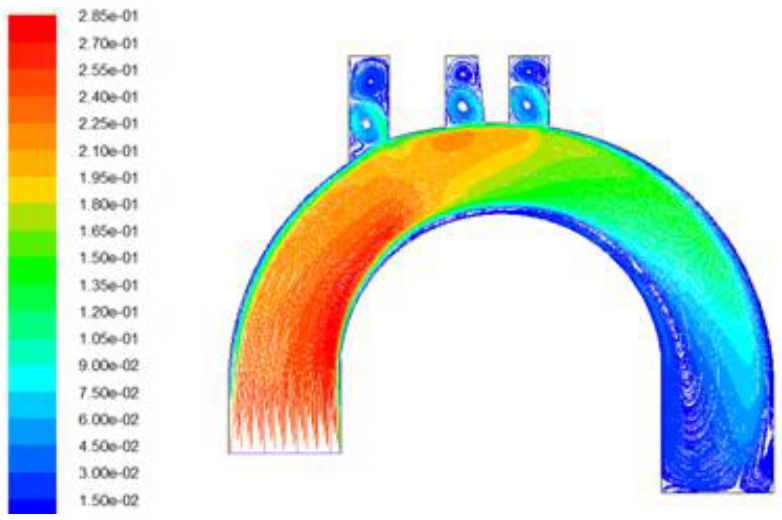

Figure 4: Velocity pathlines.

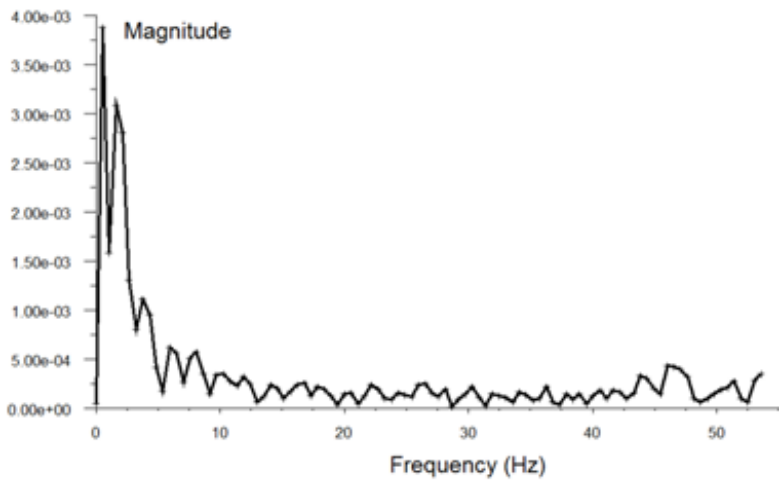

Figure 5: Velocity spectral analysis.
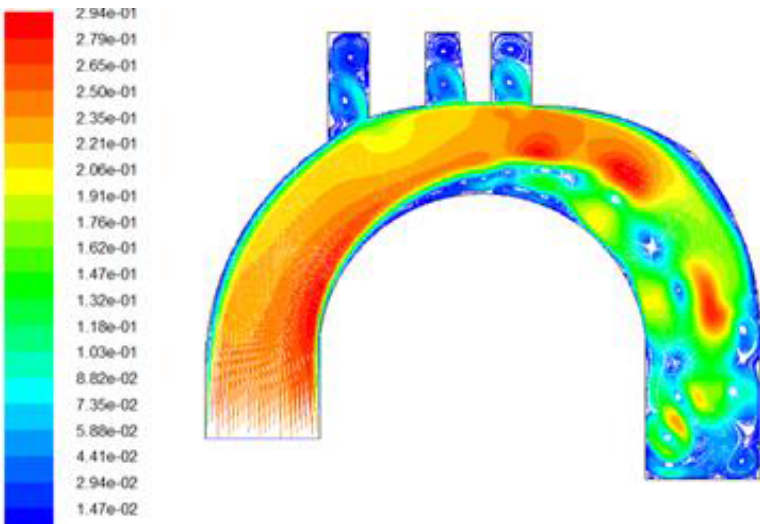

Figure 6: Velocity pathlines.

value, it is almost half than in the first case, which can be translated by the increase in the agitation of the molecules blood supporting, thus, its deceleration. The conclusion which we can draw here is that the rollers formation is the principal cause of the continuous increasing dilation of the aorta walls as the spectra values of the speed magnitude are about initial speed.

Application of a magnetic field: In this second case; we considered it useful to carry out several tests according to the possible orientations of the magnetic field and its intensity. 1er case $\mathrm{Bx}=0.02 \mathrm{~T}$ : The application of a horizontal field $\mathrm{Bx}=0.02 \mathrm{~T}$, gave the following results:

Figure 8, shows that speed preserves the same unstable behavior, except that here the size of the roller formed in the aneurismal zone is larger than the preceding one.

As for Figures 8 and 9, we see that the fundamental one and the associated harmonics have negligible magnitudes compared to the preceding cases. By projecting this case on the disorders which can take place, we can say that the consequences of this last, according to a speed review of data, are the accentuated weakening of the aorta wall or the formation of deposits (thrombus) [9].

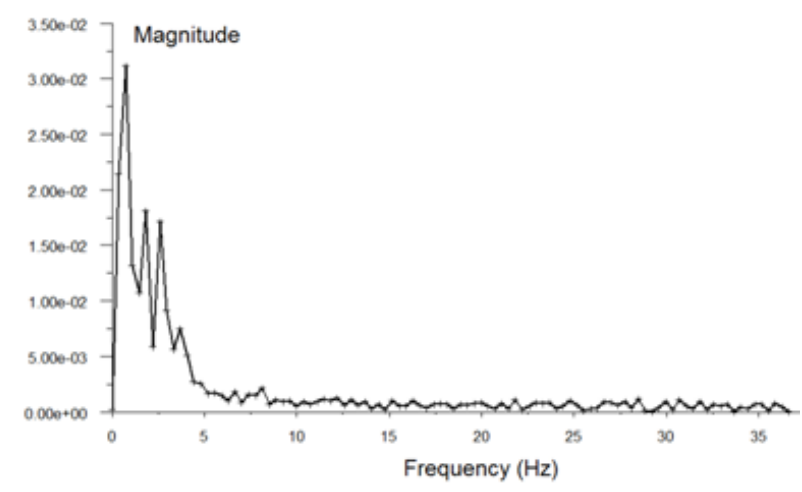

Figure 7: Velocity spectral analysis

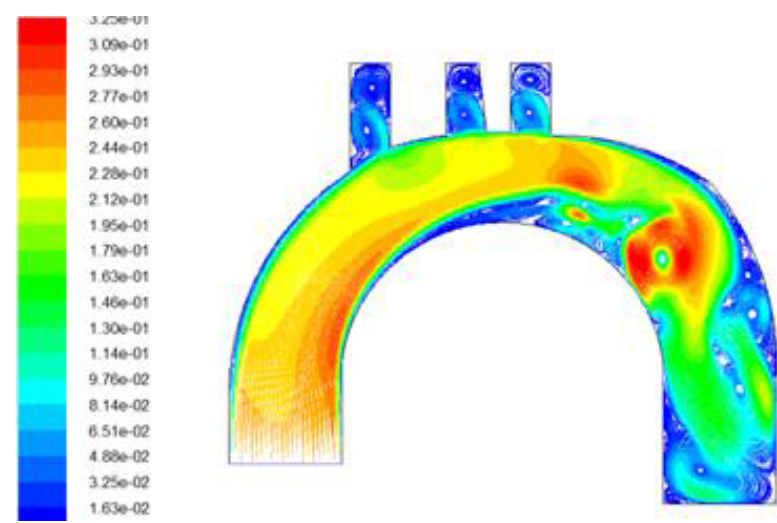

Figure 8: Velocity pathlines.

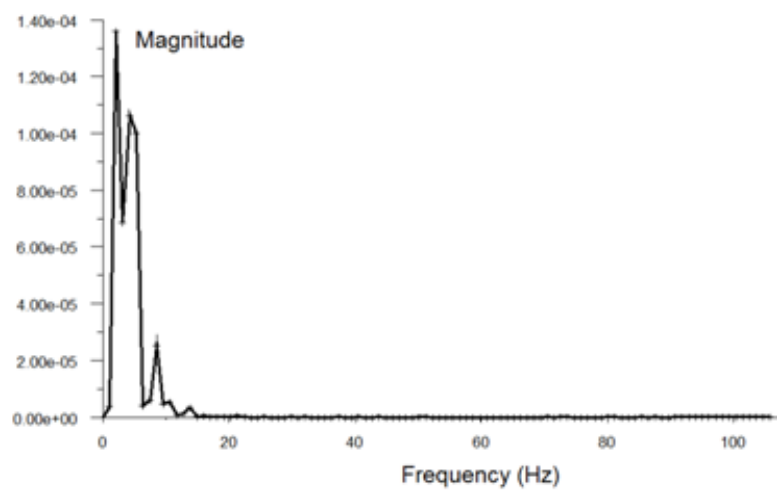

Figure 9: Velocity spectral analysis. 
Citation: Nezar M, Aggoune N, Nezar DJ, Abdessemed R, Nezar KS, et al. (2017) Effects of Magnetic Field on the Blood Flow in an Aneurismal Aorta. J Electr Electron Syst 6: 227. doi: 10.4172/2332-0796.1000227

Page 4 of 5

$\mathbf{2}^{\text {nd }}$ Case: $\mathbf{B y}=\mathbf{0 . 0 2 T}$ : The vertical orientation of the magnetic field may be considered as a particular case of this study, because, it is interested in the effect resulting from the gravitational force and the Lorentz one; on the blood flows displacement. The found results are given on Figures 10 and 11.

The interpretation of Figure 10, shows the same remarks of unsteady speed and of rollers formation in the aneurismal zone with small variations of form and at exist flow speed slightly higher than that of the top.
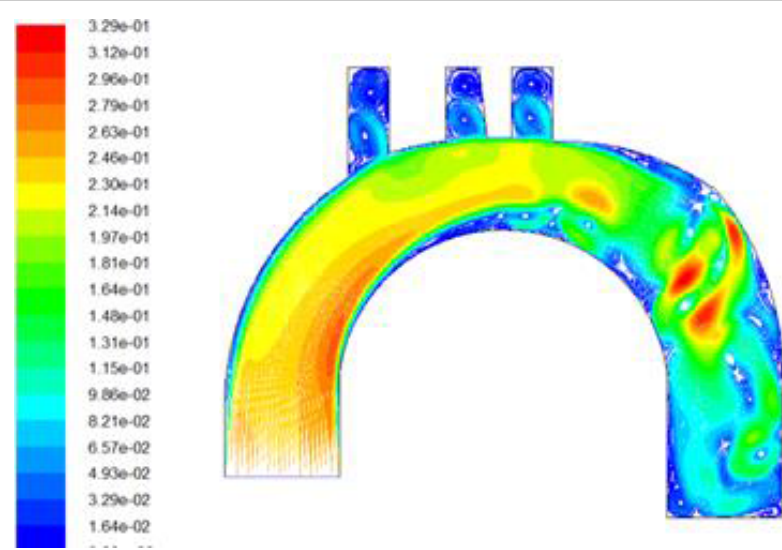

Figure 10: Velocity pathlines.

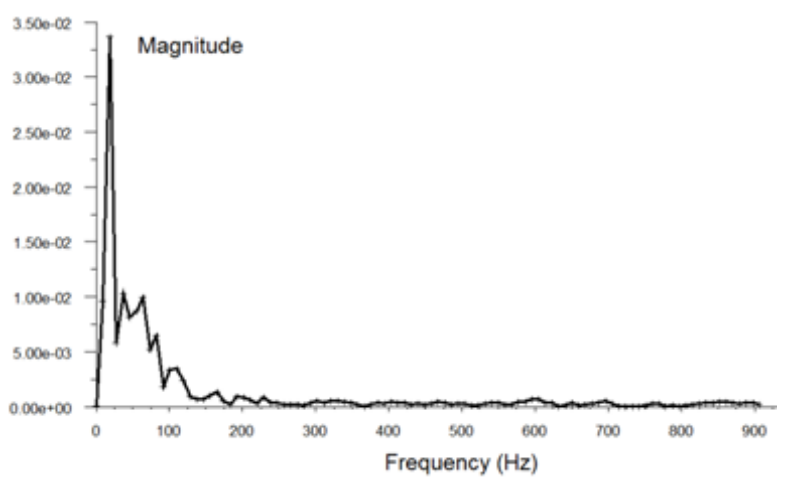

Figure 11: Velocity spectral analysis.
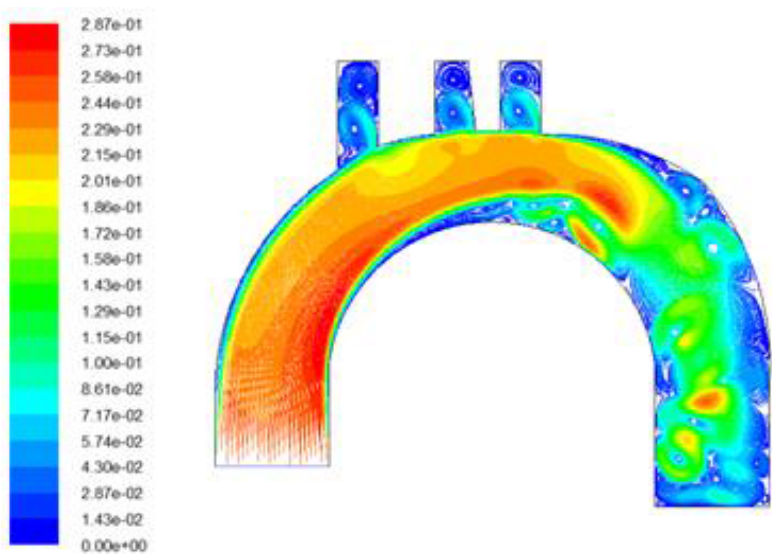

Figure 12: Velocity pathlines.

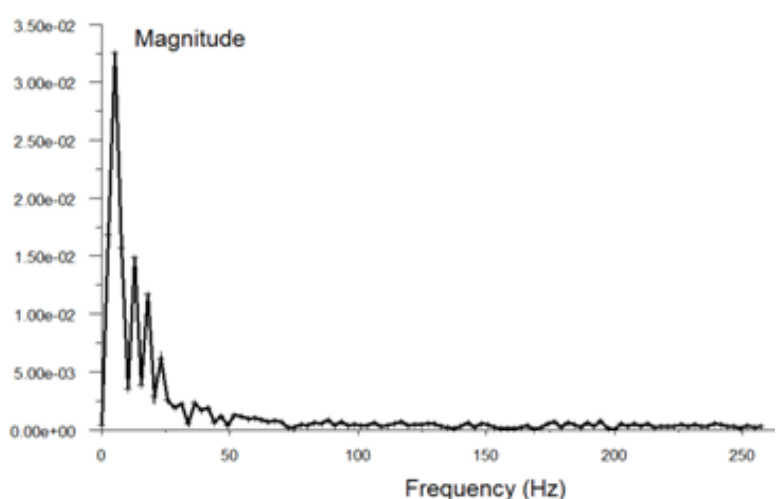

Figure 13: Velocity spectral analysis.

As for the spectral analysis, a return of fundamental towards the value of 0.035 is followed of weak harmonics which attenuate gradually [10].

$3^{\text {rd }}$ case: $\mathbf{B x}=\mathbf{B y}=1.3 \mathrm{~T}$ : The results consequential from this test show clearly that the application of the tilted field supports the progressive return towards steady speed (Figure 12) initially observed (Figure 5). But the presence of harmonic on the layout of Fourier (Figure 13), always, shows the adverse effect of the fluctuations even of low amplitudes $[11,12]$.

\section{Conclusion}

At the end of this short presentation, we can say: even if the magneto therapy has some advantages to relieve certain diseases, its application must be limited and re-examined in order to avoid all kinds of complications for the patients suffering from serious diseases like the case from the aortic aneurism, which generally carries out towards unquestionable death, while passing by pains associated with the fluctuations generated by the new channels and the formed thrombi.

\section{References}

1. HAS (2006) Evaluation des endoprothèses dans le traitement des anévrismes et des dissections de l'aorte thoracique. Service évaluation en santé publique, Service évaluation économique février, France.

2. Item 131:Anévrismes (2010) Collège des Enseignants de Médecine vasculaire et Chirurgie vasculaire. Université Médicale Virtuelle Francophone.

3. Hoang LH (2007) Contribution à la modélisation tridimensionnelle des interactions champ électromagnétique- corps humain en basses fréquences. CCSD.

4. Connes P () La viscosité sanguine, Fiche outil, Rubrique: VII / Physiologie Section: 3 / Biologie cellulaire, Numéro : A / Techniques.

5. MIA Cardiologie (2006) Item 134: L'anévrysme de l'aorte abdominaleMontpellier. Année Universitaire.

6. Lehéricy PS (2011) IRM: effets biologiques, implications de la Directive 2004/40 sur les champs électromagnétiques. SFRP.

7. Quemada D (1976) Hydrodynamique Sanguine: Hemorheologie et ecoulement du sang dans les petits vaisseaux. Hal jpa-00216431.

8. Attar A, Nataf P (2010) Anevrismes de l'aorte thoracique ascendante. Cardiologie, Presse Med 39: 26-33.

9. Scorretti R (2003) Caractérisation numérique et expérimentale du champ magnétique B.F. généré pardes systèmes électrotechniques en vue de la modélisation des courants induits dans le corps humain. Autre. Ecole Centrale de Lyon, Français.

10. Bernard $L$ (2007) Caractérisation électrique des tissus biologiques et calcul des phénomènes induits dans le corps humain par des champs électromagnétiques 
Citation: Nezar M, Aggoune N, Nezar DJ, Abdessemed R, Nezar KS, et al. (2017) Effects of Magnetic Field on the Blood Flow in an Aneurismal Aorta. J Electr Electron Syst 6: 227. doi: 10.4172/2332-0796.1000227

Page 5 of 5

de fréquence inférieur au GHz. Ecole Centrale de Lyon; Universidade federal de Minas Gerais, Français.

11. Scorretti R, Burais N (2010) Numerical dosimetry of currents induced in body by ELF magnetic fields. The International Journal for Computation and Mathematics in Electrical and Electronic Engineering.

12. Parker CB (1994) McGraw Hill Encyclopaedia of Physics. 